\title{
PROBING GALAXY EVOLUTION WITH GAMMA-RAY BURSTS
}

\author{
N.R. Tanvir ${ }^{1}$
}

\begin{abstract}
The brightest gamma-ray bursts (GRBs) would be detectable at very high redshifts and so offer a probe of star-formation and galaxy evolution into the reionization era and even beyond. Localisation of their bright afterglows pinpoints their host galaxies, however faint, and can give not only redshifts but also metallicity estimates, information on the presence of dust and molecules, and HI column densities. Statistical samples of well-observed GRBs at high redshift may therefore reveal the evolution of the global star formation rate, chemical enrichment, far-ultraviolet escape fraction and the faint-end of the galaxy luminosity function; all of which are very difficult to establish via conventional galaxy searches. To date, only a handful of $z>6$ GRBs have been identified, but their presence at $z>8$ begins to realise their potential as searchlights to illuminate the early Universe.
\end{abstract}

\section{Introduction}

Gamma-ray bursts and their afterglows are incredibly bright and are found over a huge range in redshift (e.g., Jakobsson et al. 2012). Since they are associated with the deaths of short-lived, massive stars (e.g., Hjorth et al. 2003), they pinpoint the amount and location of star-formation through cosmic time (e.g., Tanvir \& Jakobsson 2007). The power-law spectra of the afterglows provide ideal backlights for studies of abundance patterns and dust, thus offering the chance to map cosmic chemical evolution (e.g., Fynbo et al. 2006), and HI column in both the host (e.g., Jakobsson et al. 2006) and intergalactic medium (IGM). As I describe below, these probes are highly complementary to other routes to investigating early star and galaxy populations, and reionization.

1 University of Leicester, Department of Physics and Astronomy, University Road, Leicester LE1 7RH, UK 


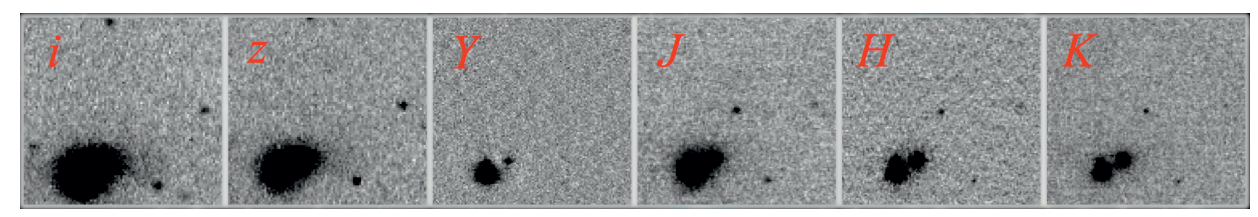

Fig. 1. Optical (GMOS-N) and infrared (NIRI) imaging of the afterglow of GRB 120923A (centred in each panel) from Gemini-North showing it to be a $Y$-band drop-out, and hence likely at $z>8$.

\section{The search for high redshift gamma-ray bursts}

Although the brightest GRBs should be detectable by Swift/BAT at very high redshifts (e.g., $z \sim 20$; Racusin et al. 2008; Bloom et al. 2009), the rate of bursts at $z>7$ is expected to be low, and finding them requires deep and rapid optical and infra-red afterglow follow-up to search for optical drop-outs and obtain redshifts. The first example to be found was GRB 090423 (Tanvir et al. 2009; Salvaterra et al. 2009), which, indeed, at $z=8.2$ was the highest spectroscopic redshift measured for any source. Remarkably, within a few days another very high redshift source was identified, although unfortunately in the case of GRB 090429B no spectroscopy was possible and we must rely on the photometric redshift of $z \sim 9.4$ (Cucchiara et al. 2011).

Since then, the rate of discoveries has remained low, and it has become clear that the majority of faint, red afterglows are in dusty systems rather than at high$z$ (e.g., Tanvir et al. 2008; Perley et al. 2009; Greiner et al. 2011) Very recently GRB 120923A was identified as another $z>8$ candidate based on optical and infrared photometry (Levan et al. 2012; see also Fig. 1); refined analysis of these data, and also spectroscopic data acquired with VLT and HST, are in progress at the time of writing. The faintness of this afterglow ( $\mathrm{AB}$ magnitude $\sim 22.5$ at discovery) confirms that follow-up with large aperture telescopes is required if we are not to miss some of these rare events.

\section{The host galaxies of high redshift GRBs}

If GRBs trace massive star formation - certainly a plausible hypothesis in the early universe - then an unbiased sample of their host galaxies should reflect the starformation weighted galaxy luminosity function. If in turn the star-formation rate is proportional to the ultraviolet (UV) luminosity, again plausible in for low-dust, low-metallicity early stellar populations, then the GRB hosts should follow the luminosity-weighted galaxy luminosity function. This argument has been applied to a sample of the hosts of six GRBs in the range $5<z<9.5$ by Tanvir et al. (2012). Even in relatively modest $H S T$ integrations, prior knowledge of the exact location of the burst (and indeed the redshift) allows us to search to very deep levels. None of the hosts was significantly detected to typical AB magnitudes limits 
of $\sim 28$, suggesting that a large proportion of high- $z$ star formation is occurring in very faint galaxies. Specifically, the conclusion was that this result is consistent with a rapidly steepening galaxy luminosity function at $z>6$, as advocated, for instance, by Bouwens et al. (2011), but marginally inconsistent with a non-evolving LF.

Interestingly, studies of the redshift distribution of GRBs (e.g., Jakobsson et al. 2012 and Robertson \& Ellis 2012 for recent discussions) also seem to require an enhanced star-formation rate at $z>3$, consistent with an increasing proportion being missed in traditional flux-limited surveys.

\section{Future developments}

The sample of high redshift GRBs identified by Swift will provide important targets for the next generation of near-infrared telescopes, including JWST and the planned $30 \mathrm{~m}$ class ground-based telescopes. Furthermore, providing there are satellites able to provide triggers in that era, spectroscopic observations of $z>7$ afterglows are likely to provide stringent constraints on the IGM neutral fraction at the position of the burst along with the HI column density and metallicity in the host (see Fig. 2). If this can be done for a sample of several tens of such events, it will allow us to map the time-line of reionization and to assess the early chemical evolution of the universe (including looking for signatures of population III nucleosynthetic yields; $c f$. . Cooke et al. 2012). Crucially, since only cases with very low host HI column density could possibly allow significant amounts of ionising UV radiation to escape from the stellar population giving rise to the GRB, the distribution of host column densities will set tight constraints on the average escape fractions for light from massive stars (e.g., see Chen et al. 2007; Fynbo et al. 2009). Unless this is considerably higher than that usually measured at lower redshifts, it is unlikely that this radiation was sufficient reionized the universe.

\section{Conclusions}

I have argued that GRBs provide a powerful complement to traditional studies of galaxy evolution, offering a route to abundances, dust properties etc. for starforming galaxies over all redshifts. Despite the sample of Swift bursts at $z>5$ to date being relatively modest in size (although new results are pending final analysis), already the searches for their hosts, and the implications their number as a function of redshift has for the global star-formation rate density, are providing important indications that the majority of high redshift star formation is occurring in galaxies below the detection limits of the deepest surveys for Lyman-break galaxies.

In the future, observations of GRBs and their hosts with next generation facilities, particularly JWST and $30 \mathrm{~m}$ class telescopes, promise to establish the properties of star-forming galaxies in the era of reionization in unprecedented detail. Swift is unlikely to remain operational into this era, thus there is very strong 


\section{$z=8.2$ simulated ELT afterglow spectrum}

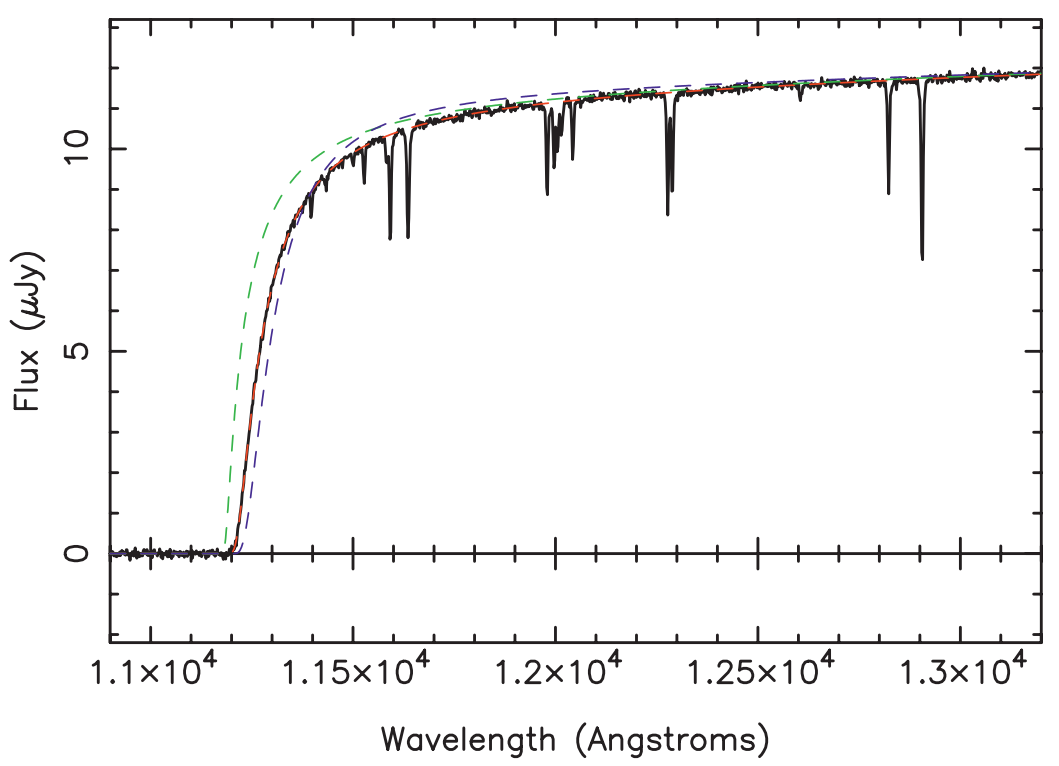

Fig. 2. Simulated spectrum (black solid line) around the Ly $\alpha$ break showing the quality of data which would be obtained with a $\sim 40 \mathrm{~m}$ telescope such as the proposed E-ELT for an afterglow with magnitude approximately the same as that obtained for GRB 090423 observed by the VLT (Tanvir et al. 2009). The host galaxy in this case was chosen to have an HI column density of $10^{21} \mathrm{~cm}^{-2}$, and a metallicity of $Z \approx Z_{\odot} / 10$, and the IGM was taken to be $100 \%$ neutral. The green dashed line shows a model with just a neutral IGM (with redshift fixed at that given by the metal lines), and the blue dashed line shows an attempt to fit a model with an ionised IGM and only absorption in the host. Neither component alone is a credible fit, illustrating that high $S / N$ data of this sort can be used to decompose IGM and host galaxy contributions (red dashed line).

motivation for development of a powerful successor mission, ideally one even better able to discover and identify very high redshift GRBs.

\section{References}

Bloom, J.S., Perley, D.A., Li, W., et al., 2009, ApJ, 691, 723

Bouwens, R.J., Illingworth, G.D., Oesch, P.A., et al., 2011, ApJ, 737, 90

Chen, H.-W., Prochaska, J.X., \& Gnedin, N.Y., 2007, ApJ, 667, L125

Cooke, R., Pettini, M., \& Murphey, M.T., 2012, MNRAS, 425, 347

Cucchiara, A., Levan, A.J., Fox, D.B., et al., 2011, ApJ, 736, 7

Fynbo, J.P.U., Starling, R.L.C., Ledoux, C., et al., 2006, A\&A, 451, L47

Fynbo, J.P.U., Jakobsson, P., Prochaska, J.X., et al., 2009, ApJS, 185, 526 
Greiner, J., Krühler, T., Klose, S., et al., 2011, A\&A, 526, 30

Hjorth, J., Sollerman, J., Møller, P., et al., 2003, Nature, 423, 847

Jakobsson, P., Fynbo, J.P.U., Ledoux, C., et al., 2006, A\&A, 460, L13

Jakobsson, P., Hjorth, J., Malesani, D., et al., 2012, ApJ, 752, 62

Levan, A.J., Perley, D.A., Tanvir, N.R., et al., 2012, GCN 13802

Perley, D.A., Cenko, S.B., Bloom, J.S., et al., 2009, AJ, 138, 1690

Racusin, J.L., Karpov, S.V., Sokolowski, M., et al., 2008, Nature, 455, 183

Robertson, B.E., \& Ellis, R.S., 2012, ApJ, 744, 95

Salvaterra, R., Della Valle, M., Campana, S., et al., 2009, Nature, 461, 1258

Tanvir, N.R., \& Jakobsson, P., 2007, RSPTA, 365, 1377

Tanvir, N.R., Levan, A.J., Rol, E., et al., 2008, MNRAS, 388, 1743

Tanvir, N.R., Fox, D.B., Levan, A.J., et al., 2009, Nature, 461, 1254

Tanvir, N.R., Levan, A.J., Fruchter, A.S., et al., 2012, ApJ, 754, 46 
